\title{
Corpus callosum infarction with cognitive dysfunction: two case reports and literature review
}

This article was published in the following Dove Press journal:

Neuropsychiatric Disease and Treatment

\author{
Jianyu Zhang \\ Yi Tang \\ Yongan Sun \\ Yingda Xu \\ Lingen Pang \\ Jiaojiao $\mathrm{Li}$ \\ Taotao Wu \\ Mingli He
}

Department of Neurology, Affiliated

Lianyungang Hospital of Xuzhou

Medical University, Lianyungang,

Jiangsu, People's Republic of China
Correspondence: Mingli He

Department of Neurology, Affiliated

Lianyungang Hospital of Xuzhou

Medical University, Tongguan North

Road No 182, Haizhou District,

Lianyungang, Jiangsu 222002,

People's Republic of China

Tel +8651885605033

Fax +86018961326515

Email lyghml@I63.com
Introduction: The incidence of corpus callosum infarction is low, and sudden cognitive dysfunction caused by corpus callosum infarction is very rare. We report two cases of acute corpus callosum infarction with sudden cognitive impairment, and the related basis, clinical manifestations, diagnosis, differential diagnosis, treatment, and prognosis of this disease are reviewed.

Cases presentation: The two patients had sudden and severe memory impairment and spatial orientation disorder. Their cognitive function scores were significantly lower, and their MRI demonstrated clear corpus callosum infarction. Through treatment, the symptoms improved significantly.

Discussion: This paper reports two cases with corpus callosum infarction with sudden cognitive impairment, and its relevant background is also reviewed, which will help doctors with the classification diagnosis of cerebral infarction and understanding of corpus callosum infarction.

Keywords: corpus callosum infarction, cognitive dysfunction, MRI, memory impairment, spatial orientation disorder

\section{Introduction}

The incidence of corpus callosum infarction is low. Giroud et $\mathrm{al}^{1}$ reported its incidence was $2.7 \%$, while Chrysikopoul et $\mathrm{al}^{2}$ reported it as $7.9 \%$. Corpus callosum infarction is often combined with other kinds of infarction, and it presents with diverse clinical manifestations, easily leading to missed or delayed diagnosis. Benefit from the progress of imaging technology, especially the diffusion-weighted imaging (DWI), has enabled the knowledge of the corpus callosum infarction to be deepened. At present, few studies focused on its clinical manifestations in the field of neurology, and sudden cognitive dysfunction caused by corpus callosum infarction is rarely reported as well. We report two cases of acute corpus callosum infarction with sudden cognitive impairment, and the related basis, clinical manifestations, diagnosis, differential diagnosis, treatment and prognosis, of this disease are also reviewed.

\section{Cases presentation Case I}

A 77-year-old male patient was admitted to our hospital on September 13, 2017 mainly because of sudden memory loss, numbness in right extremities, and lack of flexibility for the past 18 hours. The patient showed symptoms of spastic gait, a significant decline in short-term memory, and became disoriented easily after going out, but he had no dizziness, tinnitus, deafness, and disorders of consciousness. A head computed tomography (CT) scan was performed that found multiple lacunar infarcts in bilateral basal ganglia. The patient had a history of hypertension for more than 20 years, but with no detailed information on cognitive functions; he denied a history 
A

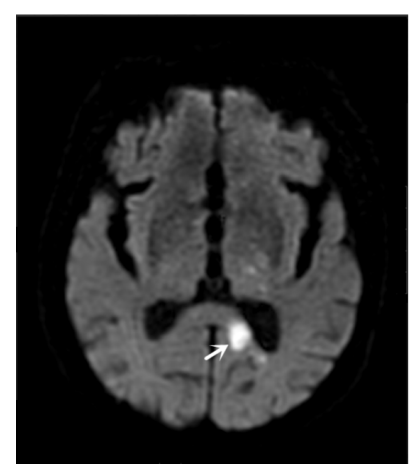

B

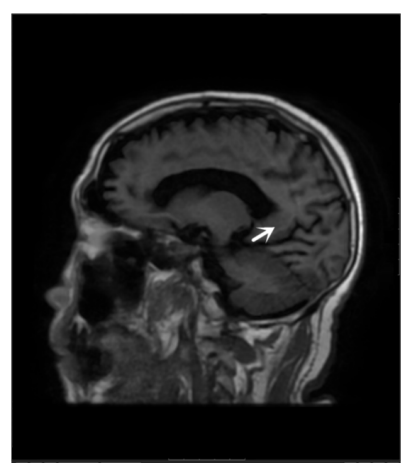

C

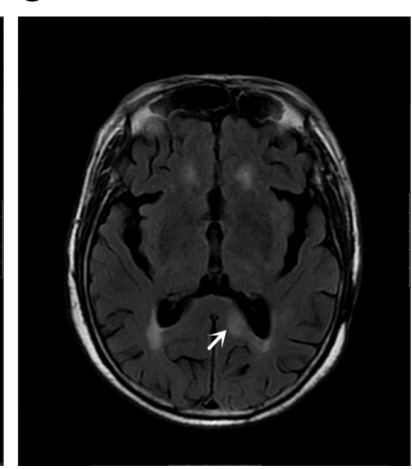

D

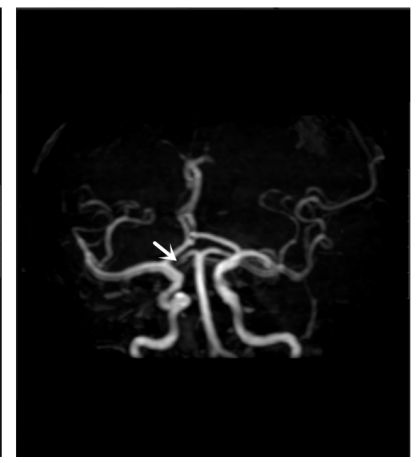

Figure I Case I magnetic resonance imaging and MRA of brain.

Note: (A) DWI, (B) TIFLAIR, (C) T2FLAIR, and (D) MRA. It showed infarction on left posterior horn of the lateral ventricle and the splenium of the corpus callosum (A-C, arrow) and occlusion of the posterior cerebral artery (D arrow).

Abbreviations: DWI, diffusion-weighted imaging; TIFLAIR, TI fluid-attenuated inversion recovery imaging; T2FLAIR, T2 fluid-attenuated inversion recovery imaging; MRA, magnetic resonance angiography.

of repeated cognitive impairment and trauma. On admission, the following examinations were performed: blood pressure, with a value of $140 / 85 \mathrm{mmHg}$, and on heart and lung testing, which showed no obvious abnormalities. Upon neurological examination, good responsiveness; obviously decreased memory, judgment, and calculation skills; and pain sensation that was markedly diminished in right extremities were observed. Doppler ultrasound of blood vessels of the neck showed multiple plaque formation on both sides of carotid bulb and the left external carotid artery. Notable changes were observed on electrocardiogram and ultrasound examination. Magnetic resonance + magnetic resonance angiography (MRA) of the brain (September 14, 2017, Figure 1A-D) showed infarction on left posterior horn of the lateral ventricle and the splenium of the corpus callosum (Figure 1A, arrow) while patchy, nodular acute infarction was observed in the occipital lobe and thalamus, associated with occlusion of the posterior cerebral artery (Figure 1D, arrow). Montreal Cognitive Assessment (MoCA) score was 12 points (Table 1). Mini-Mental State Examination (MMSE) score was 16 points. Antiplatelet therapy, hypolipidemic, and blood pressure lowering treatment were administered and the cerebral blood flow was improved after admission to hospital, which resulted in remission and discharge after 9 days. Follow-up of the patient at 1 month of discharge suggested that the memory was improved significantly (MoCA: 18 points, MMSE: 21 points).

\section{Case 2}

A 66-year-old female patient was admitted to our hospital on September 16, 2017 mainly because of numbness in right extremities and abnormal behavior for 6 days. Six days prior to admission, the patient experienced sudden weakness and numbness in right extremities, could not walk independently, and had unclear speech, dizziness, blurred vision, and nausea while vomiting several times a day. Left corpus callosum infarction was observed on head CT performed that day, followed by abnormal behavior such as eating without realizing, taking food into the mouth with both hands, long-winded speech, difficulty in identifying relatives, and so on, and so she was transferred to our department for further treatment. The patient had a history of hypertension for more than 3 years and had suffered from two episodes of vertigo in 2015 and 2016. The patient often displayed normal cognitive function and denied a history of repeated cognitive impairment and trauma. Upon admission, the following examinations were carried out: blood pressure, with a value of 122/68 $\mathrm{mmHg}$, and heart and lung testing,

Table I Case I MoCA

\begin{tabular}{lll}
\hline MoCA & First & Second \\
time & time \\
\hline Cognitive domain & & \\
\hline Visuospatial/executive & 0 & 0 \\
$\quad$ Modified trail making test & 0 & 0 \\
$\quad$ Copy of the cube & 1 & 1 \\
$\quad$ Clock drawing test & 0 & 1 \\
Naming & & \\
Memory & & \\
Attention & 2 & 2 \\
$\quad$ Digit span forward or backward & 1 & 1 \\
$\quad$ Letter A tapping test & 0 & 2 \\
$\quad$ Serial 7 subtractions & & 2 \\
Language & 2 & 0 \\
$\quad$ Sentence repetition & 0 & 2 \\
$\quad$ Letter fluency & 2 & 3 \\
Abstraction & 0 & 18 \\
Delayed recall & 3 & 12 \\
Orientation & 12 & \\
Total score &
\end{tabular}

Note: The subjects had $<12$ years of education and one more point to correct the bias.

Abbreviation: MoCA, Montreal Cognitive Assessment. 
A

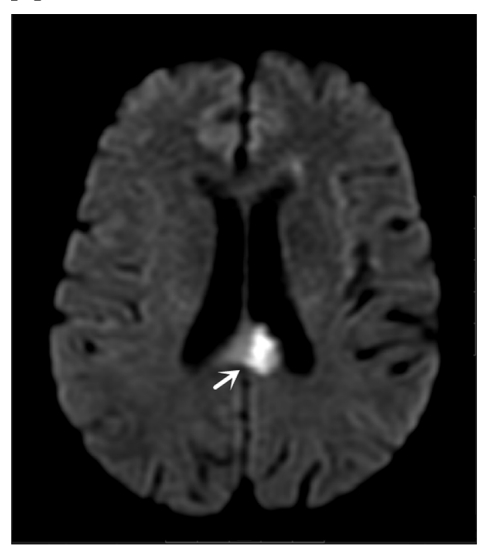

D

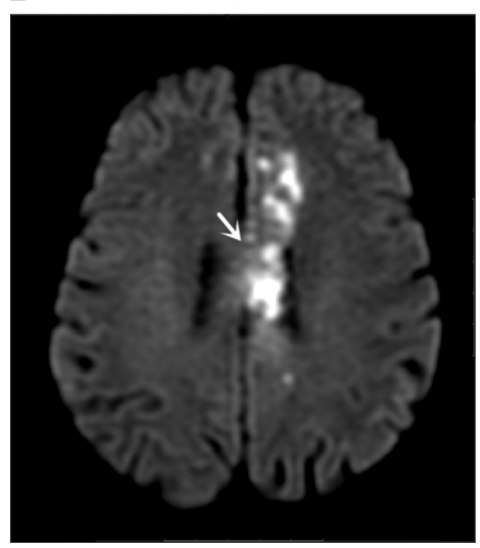

B

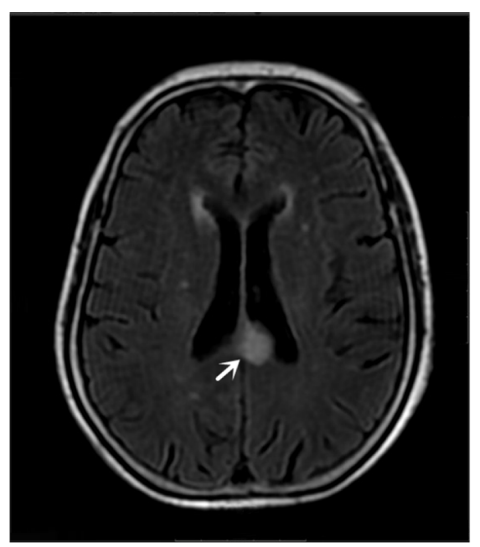

E

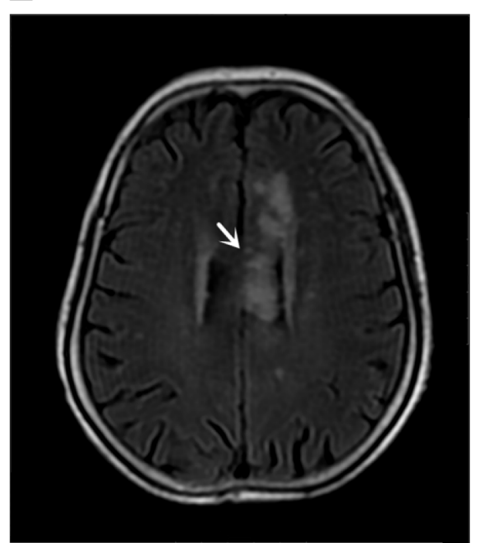

C

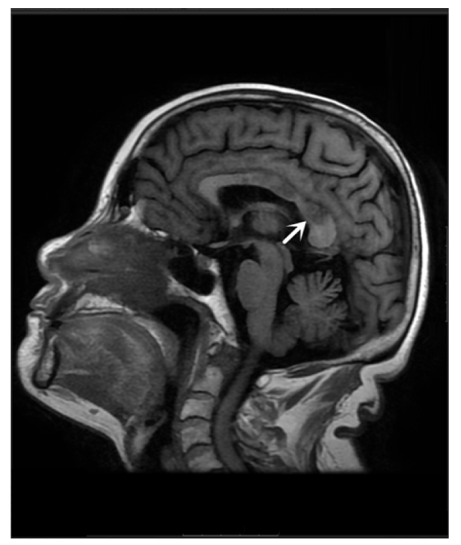

$\mathbf{F}$

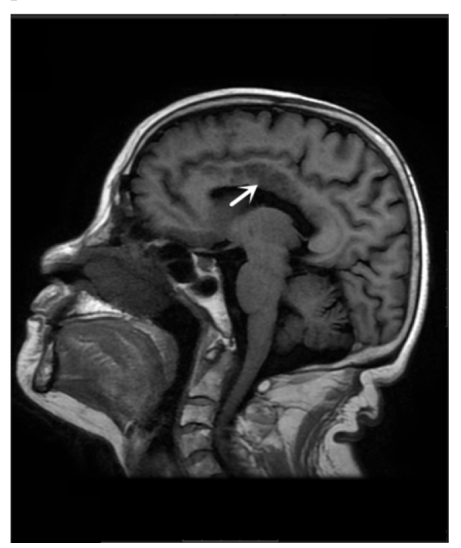

Figure 2 Case 2 magnetic resonance imaging and MRA of brain.

Note: (A) DWI, (B) T2FLAIR, (C) TIFLAIR, (D) DWI, (E) T2FLAIR, (F) TIFLAIR. It showed acute infarcts in the left centrum semiovale, involving the body of corpus callosum (A-C, arrow) and multiple lacunae of infarction (D-F, arrow).

Abbreviations: DWI, diffusion-weighted imaging; TIFLAIR, TI fluid-attenuated inversion recovery imaging; T2FLAIR, T2 fluid-attenuated inversion recovery imaging; MRA, magnetic resonance angiography.

with no obvious abnormalities detected. Upon neurological examination, she displayed good memory; obviously decreased responsiveness, judgment, and calculation skills; mild dysphonia with muscle strength graded 4th; and pain sensation that was markedly diminished in right extremities. No notable changes showed up on electrocardiogram and ultrasound examination. When magnetic resonance + MRA of brain (The First People's Hospital of Lianyungang, September 18, 2017, Figure 2A-F) was performed, acute infarcts in the left centrum semiovale, involving the body of corpus callosum (Figure 2A-C, arrow) and multiple lacunae of infarction (Figure 2D-F, arrow) were observed. MRA showed cerebral arteriosclerosis. MoCA score was 14 points (Table 2), and MMSE score was 22 points. Antiplatelet therapy, hypolipidemic, and blood pressure lowering treatment were administered. Her cognitive dysfunction was improved (MoCA: 18 points, MMSE: 24 points) at discharge from hospital after 11 days.

Statements: Both patients signed a written informed consent that includes permission to publish the case details and any accompanying images.
Table 2 Case 2 MoCA

\begin{tabular}{lll}
\hline MoCA & First & $\begin{array}{l}\text { Second } \\
\text { time }\end{array}$ \\
\hline Cognitive domain & & \\
\hline Visuospatial/executive & 0 & 0 \\
$\quad$ Modified trail making test & 0 & 0 \\
Copy of the cube & 0 & 0 \\
$\quad$ Clock drawing test & 2 & 2 \\
Naming & & \\
Memory & & 1 \\
Attention & 1 & 0 \\
$\quad$ Digit span forward or backward & 0 & 2 \\
Letter A tapping test & 0 & 1 \\
Serial 7 subtractions & & 0 \\
Language & 1 & 2 \\
Sentence repetition & 0 & 3 \\
Letter fluency & 2 & 6 \\
Abstraction & 1 & 18 \\
Delayed recall & 6 & 14 \\
Orientation & Total score &
\end{tabular}

Note: The subjects had $<12$ years of education and one more point to correct the bias.

Abbreviation: MoCA, Montreal Cognitive Assessment. 


\section{Discussion}

The corpus callosum is located between the cerebral hemispheres. It has no definite boundaries from an anatomical point of view. However, according to its connection with the cortical region, the corpus callosum is divided into four parts: rostrum, genu, body/trunk, and splenium, and it plays an important role in coordinating the signal transmission between the hemispheres. ${ }^{3,4}$ The corpus callosum is doubly supplied with blood by four vessels from the anterior and posterior circulation, the anterior communicating artery, the pericallosal artery, the posterior choroidal artery, and the posterior cerebral artery, respectively. ${ }^{5}$ Because the diameter of perforating artery in corpus callosum is small, blood from the branch arteries directly flow from the superior artery perpendicularly and emboli tend to move to the middle cerebral artery. Therefore, the blood circulation of corpus callosum is rarely blocked. Moreover, because of the rich dual blood supply, even if arterial stenosis or occlusion occurs on one side, it is compensated by the rich surrounding ramus anastomoticus. As a result, corpus callosum infarction is very rarely seen in the clinic. ${ }^{6}$

The exact function of the corpus callosum is rarely reported in the literature, because corpus callosum disorder shows no symptoms. ${ }^{7}$ The corpus callosum is one of the largest white matter fibers in central nervous system; its geniculum mainly acts as fibrous connection to the anterior cerebrum, while splenium mainly acts as the fibrous connection to posterior cerebrum. The ectocinerea associated with cognitive functions is mainly located at frontal, top, and temporal lobe of anterior cerebrum, and most of the fibers from these lobes pass through the corpus callosum. ${ }^{8,9}$ Presently, the corpus callosum is believed to manage the coordination of two hemispheres, ie, left and right cerebrum hemispheres, and plays an important role in cognitive functions, including memory and calculation capacity. After the corpus callosum becomes infarcted, the integrity of its white matter fiber tracts is damaged, and this may cause widespread disconnection between cortex and subcortical networks, which can lead to cognitive impairment. But some studies have shown that white matter fiber tracts might be partially restored after effective treatment, thereby improving impaired cognitive functions. $^{10}$

Based on the sudden onset and characteristic imaging changes observed in Case 2, with the exclusion of corpus callosum-related diseases, ${ }^{11}$ the diagnosis of corpus callosum infarction is clear. Corpus callosum infarction should be distinguished from corpus callosum tumor, MarchiafavaBignami syndrome, middle cerebral artery occlusion, multiple sclerosis of corpus callosum, and corpus callosum trauma. Corpus callosum tumor and middle cerebral artery occlusion can be ruled out based on imaging changes, while corpus callosum demyelination can be excluded based on the acute nature of disease onset and lack of any previous symptoms. Both patients in this case series have denied having a past history of similar attacks, which when combined with imaging features, helps rule out corpus callosum multiple sclerosis. Corpus callosum trauma can be ruled out because both of them denied suffering from any trauma.

Because of the important location and special function of corpus callosum, corpus callosum infarction often leads to neuropsychiatric symptoms. The abovementioned two patients showed a loss of computational ability. Case 1 also suffered from memory deterioration. A posterior infarction leads to the damage of coordination of signal exchange capacity between brain hemispheres, which results in the loss of computing ability and memory. Case 2 suffered from cognitive dysfunction, with episodes of eating without having a sense of swallowing, picking up the food into the mouth with both hands simultaneously, giving long-winded talks, as well as paroxysmal misrecognition of relatives. It may be related to the obstruction of the anterior cerebral artery, which affects the fiber of the ipsilateral frontal lobe or the contralateral frontal lobe. Moreover, it may also be associated with posterior circulation obstruction, resulting in damage to the functions of brain stem, cerebellum, and thalamus. The head MRA in Case 1 showed left posterior cerebral artery occlusion, with a combination of a history of multiple posterior circulation ischemia. The head MRA in Case 2 showed cerebral arterial diffuse sclerosis. The symptoms seen in cases all can lead to corpus callosum infarction, which is followed by edema, frontal pole impacts involving anterior cerebral artery, probably leading to occlusion, cognitive function changes, as well as abnormalities of memory and mental behavior.

The treatment of corpus callosum infarction is no different from that for general cerebral infarction, and they mainly include antiplatelet therapy, lowering of lipid and blood pressure, and reducing the relevant risk factors. Its prognosis depends on the patient's overall situation such as whether they suffered from chronic diseases in the past, the presence of any other complications, as well as infarct volume and location when infarction had occurred in other parts. In general, the main pathological change seen in corpus callosum after ischemia is demyelination, and cortical neurons are not involved; in addition, blood vessels in the corpus callosum groove merge with each other and have a developed collateral circulation and rich blood supply, which helps in timely improvement of ischemia and promotes the regeneration of myelin; therefore, the prognosis in a majority of patients 
with corpus callosum infarction is relatively better. ${ }^{12}$ The two patients mentioned in this study showed marked improvement on reexaminations with regard to MoCA and MMSE when compared to earlier results; the MMSE score after recovery in Case 1 was lower than that of Case 2, and MMSE score of Case 1 on admission was significantly lower than that of Case 2; this finding might be associated with that fact that Case 1 had been a long-term sufferer of chronic hypertension. The studies showed the hypertension might damage the cognitive functions through channels..$^{13}$

In summary, the corpus callosum infarction often combined with other parts of the infarction, resulting in a lack of specific clinical symptoms. When limb paralysis shows up in the patients associated with cognitive or mental behavior abnormalities, this disease should be considered as one potential cause. MRI, especially DWI can detect the early local lesions and help determine the condition, which can then help guide the diagnosis of cerebral infarction.

\section{Disclosure}

The authors report no conflicts of interest in this work.

\section{References}

1. Giroud M, Dumas R. Clinical and topographical range of callosal infarction: a clinical and radiological correlation study. J Neurol Neurosurg Psychiatry. 1995;59(3):238-242.
2. Chrysikopoulos H, Andreou J, Roussakis A, Pappas J. Infarction of the corpus callosum: computed tomography and magnetic resonance imaging. Eur J Radiol. 1997;25(1):2.

3. Devinsky O, Laff R. Callosal lesions and behavior: history and modern concepts. Epilepsy Behav. 2003;4(6):607-617.

4. Kahilogullari G, Comert A, Arslan M, et al. Callosal branches of the anterior cerebral artery: an anatomical report. Clin Anat. 2008; 21(5):383

5. Witelson SF. Hand and sex differences in the isthmus and genu of the human corpus callosum. A postmortem morphological study. Brain. 1989;112(Pt 3):799-835.

6. Kasow DL, Destian S, Braun C, Quintas JC, Kagetsu NJ, Johnson CE. Corpus callosum infarcts with atypical clinical and radiologic presentations. Am J Neuroradiol. 2000;21(10):1876.

7. Türe U, Yaşargil MG, Krisht AF. The arteries of the corpus callosum: a microsurgical anatomic study. Neurosurgery. 1996;39(6):1075.

8. Huang J, Auchus AP. Diffusion tensor imaging of normal appearing white matter and its correlation with cognitive functioning in mild cognitive impairment and Alzheimer's disease. Ann NY Acad Sci. 2007; 1097(1):259-264.

9. Huang J, Friedland RP, Auchus AP. Diffusion tensor imaging of normal-appearing white matter in mild cognitive impairment and early Alzheimer disease: preliminary evidence of axonal degeneration in the temporal lobe. Am J Neuroradiol. 2007;28(10):1943.

10. Nobuhara K, Okugawa G, Sugimoto T, et al. Frontal white matter anisotropy and symptom severity of late-life depression: a magnetic resonance diffusion tensor imaging study. JNeurol Neurosurg Psychiat. 2006;77(1):120.

11. Park SE, Choi DS, Shin HS, et al. Splenial lesions of the corpus callosum: disease spectrum and MRI findings. Korean J Radiol. 2017;18(4):710-721.

12. Hai C, Jianping J. Clinical study of corpus callosum infarction. J Brain Nerv Dis (Chinese). 2006;6:421-424.

13. Köhler S, Baars MA, Spauwen P, et al. Temporal evolution of cognitive changes in incident hypertension: prospective cohort study across the adult age span. Hypertension. 2014;63(2):245-251.
Neuropsychiatric Disease and Treatment

\section{Publish your work in this journal}

Neuropsychiatric Disease and Treatment is an international, peerreviewed journal of clinical therapeutics and pharmacology focusing on concise rapid reporting of clinical or pre-clinical studies on a range of neuropsychiatric and neurological disorders. This journal is indexed on PubMed Central, the 'PsycINFO' database and CAS,

\section{Dovepress}

and is the official journal of The International Neuropsychiatric Association (INA). The manuscript management system is completely online and includes a very quick and fair peer-review system, which is all easy to use. Visit http://www.dovepress.com/testimonials.php to read real quotes from published authors. 